\title{
Sources of variation in hair cortisol in wild and captive non-human primates
}

Nicolaas H. Fourie $^{\mathrm{a}, *}$, Janine L. Brown ${ }^{\mathrm{b}}$, Clifford J. Jolly ${ }^{\mathrm{c}}$, Jane E. Phillips-Conroy ${ }^{\mathrm{d}, \mathrm{e}}$, Jeffrey Rogers ${ }^{\mathrm{f}}$, Robin M. Bernstein ${ }^{\mathrm{a}, 1}$

${ }^{a}$ Department of Anthropology, Center for the Advanced Study of Hominid Paleobiology, The George Washington University, 2110 G Street NW, Washington, DC 20052, USA

${ }^{b}$ Center for Species Survival, Smithsonian Conservation Biology Institute, Front Royal, VA 22630, USA

${ }^{c}$ Department of Anthropology, New York University, Rufus D. Smith Hall, 25 Waverly Place, New York, NY 10003, USA

${ }^{d}$ Department of Anatomy and Neurobiology, Washington University School of Medicine, 660 South Euclid Avenue, Saint Louis, MO 63110, USA

${ }^{e}$ Department of Anthropology, Washington University in St. Louis, McMillan Hall, Room 112, One Brookings Drive, St. Louis, MO 63130, USA

${ }^{f}$ Human Genome Sequencing Center and Department of Molecular and Human Genetics, Baylor College of Medicine, 1 Baylor Plaza, Houston, TX 77030, USA

*Corresponding author. Present address: Biobehavioral Branch, Division of Intramural Research, National Institutes of Health, Bethesda, MD 20892, USA. Phone: +1 (301) 451-1676, fax: +1 (301) 480-8286.

E-mail address: fourienh@mail.nih.gov (N.H. Fourie).

${ }^{1}$ Present address: Department of Anthropology, University of Colorado Boulder, 1350 Pleasant St., Boulder, CO 80309, USA. 


\section{ABSTRACT}

Hair cortisol analysis is a potentially powerful tool for evaluating adrenal function and chronic stress. However, the technique has only recently been applied widely to studies of wildlife, including primates, and there are numerous practical and technical factors that should be considered to ensure good quality data and the validity of results and conclusions. Here we report on various intrinsic and extrinsic sources of variation in hair cortisol measurements in wild and captive primates. Hair samples from both wild and captive primates revealed that age and sex can affect hair cortisol concentrations; these effects need to be controlled for when making comparisons between individual animals or populations. Hair growth rates also showed considerable inter-specific variation among a number of primate species. We describe technical limitations of hair analyses and variation in cortisol concentrations as a function of asynchronous hair growth, anatomical site of collection, and the amount and numbers of hair/s used for cortisol extraction. We discuss these sources of variation and their implications for proper study design and interpretation of results.

Keywords: Cortisol; Hair cortisol analysis; Stress; Primates; Chronic stress 


\section{Introduction}

The hypothalamic-pituitary-adrenal (HPA) axis (Nelson, 2005) is the principal endocrine axis responsible for maintaining homeostasis in the face of an external stressor. Cortisol is one of the principal hormones produced by this axis. Both psychosocial and physical stressors can result in elevated cortisol concentrations. The short-term effect of cortisol is adaptive but if chronically maintained at high levels it can have negative health effects (Sapolsky, 1992a, 2004, 2005; de Kloet et al., 1999; Chrousos, 2000). For this reason cortisol is often used as a measure of the physiological costs associated with particular social, biological (e.g. reproductive) and/or environmental conditions (e.g., Sapolsky, 1992b; Saltzman et al., 1994; Gesquiere et al., 2005; Kirschbaum et al., 2009). Shortterm or acute fluctuations in cortisol are measured in substrates such as blood, saliva, urine or feces. These substrates reflect HPA activity over a period of minutes to days (Bahr et al., 2000). Measurement of short-term fluctuations in cortisol is useful in the study of the immediate reactions of animals to external stimuli (social/environmental) and the associated physiological consequences or costs (e.g., Sapolsky, 1992a,b, 2004, 2005). For insights into long-term or chronic stress multiple samples need to be collected over a defined period of time. Such approaches are useful in assessing cross-sectional or population level responses to ecological or environmental shifts or assessing the physiological consequences and costs of sustained stress (Pearson et al., 2015).

Alternatively, hair is a reliable archive of chronic cortisol secretion (Davenport et al., 2006; Sauve et al., 2007; Accorsi et al., 2008; Thomson et al., 2010). As a means of measuring chronic stress in animals, hair cortisol analyses are finding wider application in both captive- and field-based research (e.g., Davenport et al., 2006; Accorsi et al., 2008; Koren et al., 2008; Bechshøft et al., 2010; Fairbanks et al., 2011; Carlitz et al., 2015; Fourie et al., 2015a,b; also see Russell et al., 2012 for review). The interest in hair as an archive of endocrine activity derives from the various unique features of hair that offer advantages to researchers interested in chronic stress compared to more traditional substrates (e.g., blood, stool, urine, saliva) used to determine cortisol levels. Cortisol is 
incorporated into the hair shaft through two principal mechanisms. The free, unbound fraction of cortisol is incorporated during growth of the follicle (Cone, 1996). In addition, sweat and sebum excreted by glands on the skin contain cortisol which can become incorporated into the hair shaft (Anielski, 2008). Consequently, hair samples offer a way to measure the average cortisol levels accumulated over the lifetime of the hair. Evidence suggests that hair can also be analysed serially to reveal temporal changes in cortisol levels (e.g., Kirschbaum et al., 2009; Webb et al., 2010). The time-averaged nature of the cortisol signal in hair reflects the basal hormonal phenotype (long-term time-averaged cortisol secretion) of an individual (Davenport et al., 2006). While other sample types can be used to estimate the basal phenotype and provide high resolution longitudinal insights, multiple sampling over an extended period of time is required. On the other hand, the basal phenotype can be estimated using a single sample when using hair. Another advantage is that hair appears to be a stable environment for steroids, so samples can be shipped and stored at ambient conditions and remain biologically relevant for long periods of time (e.g. >1000 years; Webb et al., 2010, 2015). Hair can also be collected using minimally invasive techniques, such as using a variety of hair traps, from both captive and wild animals (Valderrama et al., 1999). The extraction of steroids, particularly cortisol, from hair is relatively simple and concentrations can be quantified using standard immunosorbent assay techniques (e.g., Koren et al., 2002; Davenport et al., 2006). These properties make it an attractive substrate for assessing chronic/long-term adrenal function in mammals under a variety of conditions.

Various factors may influence the cortisol measured in hair. We know from previous reports that hair colour (Bennet and Hayssen 2010; Yamanashi et al., 2013), hair type (Macbeth et al., 2010), anatomical region from which it was sampled (Sauve et al., 2007; Macbeth et al., 2010; Terwissen et al., 2013; Yamanashi et al., 2013; Carlitz et al., 2014, 2015), as well as preparation techniques (Davenport et al., 2006) can influence hair cortisol concentrations. Here we examine sources of variation in hair cortisol, some of which could be considered sources of error in the accurate 
estimation of hair cortisol (e.g., sample preparation, sample weight and number of hairs) and others which reflect real biological variation (variation in hair cortisol by anatomical site, age, sex, hair growth rate) from data accumulated over the course of several studies in wild and captive primates (Fourie and Bernstein, 2011; Fourie et al., 2015a,b). We describe and consider how they impact the interpretation of individual hair cortisol levels, as well as cross-sectional/population level comparisons. These findings have important practical implications for the application of the technique and appropriate study design for both captive and wild animals, and inform the inferences that can be drawn from results given practical constraints of sample collection and study sample composition.

\section{Materials and methods}

\subsection{Sample collection}

Hair from wild yellow baboons (Papio cynocephalus; $\mathrm{n}=5$ ) was collected from animals anesthetized in the field in the course of unrelated research in the Mikumi National Park, Tanzania (PhillipsConroy et al., 1987). Hair samples from chacma baboons (Papio ursinus; $\mathrm{n}=14$ ) from Loskop Dam Nature Reserve, Suikerbosrand Nature Reserve and the city of Tshwane (South Africa) were obtained from dead animals found by park rangers, or were provided by wildlife veterinarians attending captured animals. Hair samples from captive Guinea baboons (Papio papio; $\mathrm{n}=26$ ) collected during annual veterinary exams were provided by the Museum de Besançon, France. Hair samples from other primate taxa, including Allen's swamp monkey (Allenopithecus nigroviridis; $\mathrm{n}=$ 2), golden-bellied mangabey (Cercocebus chrysogaster; $\mathrm{n}=3$ ), gelada baboon (Theropithecus gelada; $\mathrm{n}=1$ ), western lowland gorilla (Gorilla gorilla; $\mathrm{n}=3$ ), yellow-cheeked crested gibbon (Hylobates gabriellae; $\mathrm{n}=1$ ), and siamang (Symphalangus syndactylus; $\mathrm{n}=1$ ) were provided by the San Diego Zoo, also collected during annual veterinary exams. Hair from a white-faced saki 
(Pithecia pithecia; $\mathrm{n}=1$ ) was collected opportunistically from a deceased animal from the Santa Ana

Zoo upon necropsy.

Exact ages were not available for the wild animals so age determinations were based on molar dental eruption schedules, based on an age class system devised by Phillips-Conroy and Jolly (1988) for Papio baboons: infants - age class $1=$ no permanent molars showing $(<2$ years $)$; young juveniles age class $2=$ first molar showing $(2-4$ years); sub-adults - age class $4=$ third molar showing $(4-8$ years) (older juveniles and sub-adults were combined - after Beehner et al., 2009); adults - age class $5=$ all teeth in full occlusion ( $>8$ years). The birth dates of the captive animals were usually known and accurate calendar ages could be calculated for each animal.

Hair from 5 female yellow baboons was collected from three anatomical regions: the base of the tail, thigh and deltoid areas. Hair of all other individuals from both wild and captive populations was sampled from the inter-scapular region. Once received, samples were stored in labelled paper envelopes at ambient laboratory temperature.

Hair from the wild animals (and the captive Guinea baboons) was used to examine the effects of age, sex and the anatomical origin of the hairs on hair cortisol variation. Hair from the captive animals was used to assess hair growth rates and the hair from the saki monkey was used to assess the effect of sonication on extraction efficiency, and the relationship between sample weight and the number of hairs per unit weight on the accuracy with which hair cortisol concentration could be determined.

\subsection{Sample preparation and extraction}

\subsubsection{Cortisol extraction method}

Hair samples (10 mg) were weighed into labelled $5 \mathrm{ml}$ borosilicate $12 \mathrm{~mm}$ x $75 \mathrm{~mm}$ tubes and twice washed in $3 \mathrm{ml}$ isopropanol for $3 \mathrm{~min}$ to remove exogenous contaminants, sweat and sebum (Davenport et al., 2006), and dried in a fume hood for $24 \mathrm{~h}$. Once dry, the samples were minced into 1-2 mm pieces with surgical scissors, and $4 \mathrm{ml}$ of methanol was added. The scissors were rinsed into 
116

1

1 ใุ 7

4
158

6

119

120 11

121 13

$1 \frac{12}{15}$

each tube to ensure that no hair material was lost. The minced samples were then incubated in a sonicating water bath at $50 \mathrm{~Hz}$ at $55^{\circ} \mathrm{C}$. Aliquots of saki monkey hair $(10 \mathrm{mg})$ were incubated in the sonicating hot water bath for $0,60,120,240$, or $360 \mathrm{~min}$ to evaluate the effect of differing incubation times on cortisol extraction from hair. All samples were then incubated on a plate-shaker for the remainder of the 24-h extraction period. The tubes were then centrifuged at $2500 \times \mathrm{g}$ for $5 \mathrm{~min}$, and a $3.5 \mathrm{ml}$ aliquot of the supernatant was pipetted into labelled polypropylene tubes and dried under a stream of air in a fume hood. The extract was reconstituted and dried in successively smaller volumes of methanol $(2 \mathrm{ml}, 1 \mathrm{ml}$ and $500 \mu \mathrm{l})$ leaving the dry extract at the bottom of the tube. The dry extract was reconstituted in $500 \mu \mathrm{l}$ of phosphate buffer provided in the assay kits and stored at $20{ }^{\circ} \mathrm{C}$.

\subsubsection{Sample size and composition}

In order to assess the effect of the number of hairs of which a sample consists on the accuracy of estimates of the cortisol "phenotype" of an individual, $3 \mathrm{mg}, 10 \mathrm{mg}$ and $20 \mathrm{mg}$ hair samples from a saki monkey were processed as described above. The number of hairs in a $1 \mathrm{mg}$ sample was counted and was used as a basis for extrapolating the number of hairs for other weights. The length of five individual hairs was also measured and averaged to get an idea of the relationship between hair length and weight for this species.

\subsection{Hormone analysis}

Hair cortisol concentrations were quantified using an enzyme-linked immunosorbent assay (ELISA) designed to quantify free unbound cortisol in saliva (ALPCO Diagnostics, Salem, NH, USA). Manufacturer reported cross-reactivity as follows: cortisol $=100 \%$, prednisolone $=13.6 \%$, corticosterone $=7.6 \%$, deoxycorticosterone $=7.2 \%$, progesterone $=7.2 \%$, cortisone $=6.2$, deoxy cortisol $=5.6 \%$, prednisone $=5.6 \%$, dexamethasone $=1.6 \%$. The sensitivity of this assay was 1 
$\mathrm{ng} / \mathrm{ml}$. Reconstituted samples $(50 \mu \mathrm{l})$ were analyzed in duplicate, and results were converted from $\mathrm{ng} / \mathrm{ml}$ of solute to $\mathrm{ng} / \mathrm{mg}$ of dry hair weight. Immuno-specificity of the ALPCO salivary cortisol ELISA for endogenous cortisol extracted from non-human primate hair (50 mg sample pools) was demonstrated by showing parallelism between dilutions of cortisol standard $(0,1,3,10,30$ and 100 $\mathrm{ng} / \mathrm{ml}$ ) and hair cortisol extracts (neat, 1:2, 1:4, 1:8, 1:16, 1:32) for all the taxa examined. Recovery of cortisol standard added to extracts was greater than $90 \%$ for all taxa.

\subsection{Hair growth rates}

After collection of hair samples from animals housed at the San Diego Zoo (Allen's swamp monkey, golden-bellied mangabey, gelada baboon, western lowland gorilla, yellow-cheeked crested gibbon, Siamang), zoo personnel visually inspected individuals on a regular basis and the date of full hair regrowth was recorded. Zoo personnel reported full regrowth when they could no longer distinguish the shaved area from adjacent unshaved areas. An estimated hair growth rate for each species was determined based on measured mature hair lengths (average of the measurement of 5 individual hairs) and the time that had lapsed between the day of shaving and the day that full regrowth was reported.

\subsection{Data analysis}

Differences in the amount of cortisol extracted from hair using different sonication times were tested using a Kruskal-Wallis (KW) test, followed by post hoc pairwise comparisons using Gao's nonparametric multiple test procedure (Gao et al., 2008). Inter-aliquot variability was described using basic descriptive statistics, including calculation of the coefficient of variation for each aliquot size category. Hair regrowth rates were similarly examined using descriptive statistics. Variability in hair cortisol from hair sampled across the different body regions in the 5 yellow baboon females was tested using a paired $t$-test. Regression analysis was used to examine the relationship between hair 
cortisol and age, and Lowess curves were fitted to the data to visually describe trends in the data.

Kruskal-Wallis and post hoc pairwise comparisons were used to assess the variation in hair cortisol across age categories and between sexes in the Guinea and chacma baboon samples. To improve statistical power of group analyses, age classes 3 and 4 were pooled for the Guinea baboon samples. Statistical significance was set to $\mathrm{p}<0.05$ and the software packages Statistica v12 (StatSoft, Tulsa, OK, USA) and the R package nparcomp v2.6 (Konietschke, 2015) in R v3.2 (R Development Core Team, 2015) were used to perform all statistical analyses.

\section{Results}

\subsection{Technical sources of variation}

\subsubsection{Incubation in sonicating hot water bath}

The amount of cortisol extracted from saki monkey hair under the various experimental conditions differed ( $\mathrm{KW}$ test: $\left.\mathrm{H}_{4,44}=25.17, \mathrm{p}<0.0005\right)$. All extractions yielded up to 1.5 times more cortisol when incubated in a sonicating hot water bath than when incubated at ambient temperature (Gao's test: all $\mathrm{p}<0.05$ ), and the yield of cortisol increased with duration of sonication up to $240 \mathrm{~min}$ (Gao's test: 120 min vs. $240 \mathrm{~min} ; \mathrm{p}=0.01$ ), after which there was no significant change in yield (Gao's test: 240 min vs. 360 min; p > 0.05; Fig. 1).

\subsubsection{A model example of hair sample composition: hair of the white-faced saki}

Hairs sampled from the inter-scapular region of the white-faced saki were $6.13 \pm 0.37 \mathrm{~cm}$ (mean \pm standard deviation) long $(n=5) .7$ of these hairs with an average length of approximately $6 \mathrm{~cm}$ weighed approximately $1 \mathrm{mg}$. Thus, by extension, a $3 \mathrm{mg}$ sample contains approximately 21 hairs, a $10 \mathrm{mg}$ sample contains approximately 71 hairs, and a $20 \mathrm{mg}$ sample contains approximately 144 hairs. The inter-aliquot hair cortisol coefficient of variation decreased from $40 \%$ for $3 \mathrm{mg}$ samples (n $=7)$ to $13 \%$ in $10 \mathrm{mg}$ samples $(\mathrm{n}=13)$ and $9 \%$ in $20 \mathrm{mg}$ samples $(\mathrm{n}=5)($ Fig. 2$)$. 


\subsubsection{Anatomical region}

No differences in cortisol concentrations were observed between hair collected from the base of the tail and the shoulder, or the thigh and the shoulder of yellow baboons ( $p>0.05)$. However, hair sampled from the thigh yielded significantly higher cortisol concentrations than hair from the base of the tail (paired t-test, $\mathrm{n}=5, \mathrm{t}(4)=-3.16, \mathrm{p}=0.03$; Fig. 3).

\subsection{Intrinsic sources of variation}

\subsubsection{Hair growth rates}

Rough estimates of hair growth rates based on observational data recorded by animal keepers at San Diego Zoo suggest considerable inter-specific variation in hair growth rates (Table 1). Hair growth rates varied from $0.7 \pm 0.1 \mathrm{~cm}$ per month in yellow-bellied mangabeys $(\mathrm{n}=3)$ to $3.5 \mathrm{~cm}$ per month in the single gelada baboon sample. Time to full regrowth varied from 2.8 months in western lowland gorillas $(n=3)$ to 7.1 months in yellow-bellied mangabeys. Two gorillas had surprisingly short hair $(4.3 \pm 0.4 \mathrm{~cm})$, which took approximately 3.7 months to regrow to full length. The third individual had much longer hair $(13.9 \pm 1.3 \mathrm{~cm})$, which appeared to grow more rapidly $(5.8 \mathrm{~cm}$ per month $)$ and reached full length after only 2.4 months. The long hair and rapid regrowth of one individual accounts for the high standard deviations in hair growth parameters which we reported for this species.

\subsubsection{Age and sex effects}

Multiple regression analysis confirmed a negative relationship $(\beta=-0.47, \mathrm{t}(23)=-2.34, \mathrm{p}=0.05)$ between age and hair cortisol levels in the captive Guinea baboon samples due to the overall higher concentrations in infants (KW test: $\mathrm{H}_{1,26}=8.86, \mathrm{p}=0.003$; Fig. 4) compared to other age groups. There were no differences in cortisol concentrations among the older age classes $(p>0.05)$. No 
216

1

2द⿱ 7

2\$8

219

นวิ์

11

221

13

这2

16

223

18

${ }_{2} 24$

21

225

23

326

26 28

298

30

319

relationship was found between hair cortisol level and sex in Guinea baboons ( $p>0.05)$ (note that few males at older age categories were available for this comparison).

There was also a negative relationship between age and hair cortisol concentrations in the chacma baboon samples $(\beta=-0.615, \mathrm{t}(10)=-4.511, \mathrm{p}<0.005)$. One male $(\mathrm{Tsh} 1-$ see Fig. 5$)$ was identified as an outlier ( $>2$ times the outlier coefficient of 1.5), and exhibited hair cortisol concentrations (4.05 $\mathrm{ng} / \mathrm{mg}$ ) that were at least twice as high as those observed in any other chacma baboon for which we had data. This individual was therefore excluded from comparisons between ages and sex classes in the chacma sample. In chacma baboons, hair cortisol levels were higher in females than males $(\beta=$ $0.524, \mathrm{t}(10)=4.847, \mathrm{p}<0.005)$.

\section{Discussion}

Using samples collected from wild and captive non-human primates, we explored intrinsic and extrinsic/technical sources of variation in hair cortisol measurements, which have implications for the applicability of this technique for studying adrenal activity and stress. Some of the variability was due to extraction methodology (i.e., effects of sonication). We found that incubating samples in a sonicating hot water bath may present a viable alternative to the use of expensive milling equipment to improve extraction efficiency. Including even $60 \mathrm{~min}$ of sonication in a hot water bath significantly increased the extraction rate, compared to shaking incubation at ambient temperature alone. The amount of cortisol extracted did not increase after $240 \mathrm{~min}$ of incubation in the sonicating hot water bath, and appeared to decrease at the longer incubation interval (360 min), although this decrease was not statistically significant. We decided to limit the sonicating hot water bath incubation step to 240 min to avoid possible degradation of cortisol due to prolonged exposure to high temperatures and/or sonication. Additional experiments are required to examine whether heat or sonication or the combination of both is the main driver of the increased extraction rate. Published protocols for the extraction of cortisol from hair require the reduction of the substrate to small 
particles by cutting (e.g., Sauve et al., 2007) or milling (e.g., Davenport et al., 2006) in order to maximize the cortisol extracted. It is known that sonication, specifically sonication in a hot water bath, can significantly improve the extraction of endobiotics from substrates with similar physicochemical properties to hair (Schinor et al., 2004). It is also used in doping research to extract anabolic steroids and their metabolites from hair (e.g., Gaillard et al., 2000; Anielski, 2008). It is thought that sonication accelerates the transfer of compounds into solution by disrupting the matrix (Melecchi et al., 2002). Some researchers do include sonication as a brief step ( 30-45 min), using standalone sonicators, in cortisol extractions from hair (Koren et al. 2002; Kalra et al., 2012).

The size of the hair sample aliquots had an effect on measured cortisol, with samples consisting of fewer hairs having greater inter-aliquot variability in hair cortisol measured. Our results suggest that approximately $10 \mathrm{mg}$ (70 hairs) are needed per aliquot to accurately determine mean hair cortisol concentrations for the investigated animal (a white-faced saki), and perhaps for any primate with hair of similar length, thickness and growth pattern. Samples consisting of only a few hairs do not yield an accurate estimation of mean hair cortisol ("cortisol phenotype" - Davenport et al., 2006) for this animal. As more hairs are included in a sample, the accuracy of the estimation of mean hair cortisol improves. This pattern of sample composition-dependent variation in hair cortisol is consistent with a scenario where different hairs are in different phases of growth (e.g., anagen, catagen or telogen) and reflect different periods of endocrine activity (asynchronous growth) (Harkey, 1993). This would not be expected if hair growth was synchronous, as all hairs would record an identical period of endocrine activity and analysis of individual hairs or aliquots of varying composition (i.e., number of hairs) would yield a low and roughly equivalent inter-hair coefficient of variation (CV). Our results reflect the former scenario, though the effects of variation in hair thickness, length and local anatomical effects (e.g., Sauve et al., 2007) are also likely to affect hair cortisol variation in both synchronous and asynchronously growing hair populations. This suggests that as in humans, hair growth in the model taxon used for this experiment (white-faced saki monkey) is asynchronous. We 
suggest, based on these results, that if the focus of a study is to make inter-individual comparisons, relatively large samples (i.e., greater number of hairs) may be necessary in order to accurately characterize the "cortisol phenotype" of an individual animal. If a study aims to make broad population-based comparisons, a larger error in the estimation of individual hair cortisol may be tolerated and smaller samples (i.e., fewer hairs per sample) could potentially be used. Dimensions and properties of the hair itself act as limiting factors for determining optimal sample size. In large primates with long and thick hair, a single hair may yield enough cortisol to be measured by ELISA, but would yield a poor estimation of the true chronic cortisol for that individual animal because it is not representative of the larger hair population. For a small primate (e.g., a callitrichid), a large number of individual hairs would be needed to make up a sufficient weight to yield enough cortisol for measurement; by default, in this situation the individual's hair population would be well sampled and able to offer a good reflection of the animal's true chronic cortisol. In order to strike the appropriate balance it is important for researchers to conduct a preliminary investigation on the properties of the hair of the taxon they are studying to determine the appropriate sampling strategy. For example, 10-20 mg samples (e.g., 50-100 hairs) may be appropriate when studying mediumsized primates, such as saki monkeys or baboons, but individual hairs ( $10 \mathrm{mg}$ each) collected from a gorilla nest may not be sufficient to make detailed inter-individual comparisons between animals, although they could be informative as part of a large cross-sectional regional data set comparing different populations.

We found that variation in hair cortisol concentrations may be affected by the anatomical region from which a sample is taken. Variability across body regions in hair cortisol concentrations has been reported in humans (Sharpley et al., 2010a, b), grizzly bears (Macbeth et al., 2010), and chimpanzees and orang-utans (Carlitz et al., 2014, 2015), and emphasizes the importance of controlling for anatomical region when sampling hair. This may not always be possible, especially when hair is collected opportunistically or via remote sampling methods, such as hair traps. In such 
cases, anatomical variability in hair cortisol should be examined in the study species and considered in the study design. Depending on the nature of the questions posed it may be possible to accommodate the variation resulting from differences in anatomical region in the study design.

Although sample sizes were small, and completion of hair growth was assessed by observation only, to our knowledge our findings on hair growth rate across several non-human primate species are the first to be reported. However, the estimated growth rates we report should be interpreted with caution as this methodology is imprecise. More precise experiments should be conducted to confirm the observations we report. The importance of these results is that they emphasize that considerable variation in hair growth rates may exist within and between species. A better understanding of this variability is needed to interpret individual hair cortisol levels in the context of seasonal and other environmental effects, as well as when making inter-individual/species comparisons.

We found differences in hair cortisol as a function of age in both chacma and Guinea baboons. Infants and young juveniles had higher hair cortisol levels than other age groups. Similar age-related patterns in hair cortisol have been observed in both olive baboons (Papio anubis) and vervet monkeys (Chlorocebus aethiops) (Fourie and Bernstein, 2011; Laudenslager et al., 2012; Fourie et al., 2015b). Such negative correlations between cortisol and age reflect ontogenetic patterns of adrenocortical function, and have been found in other studies using cortisol measured in plasma and feces collected from captive and wild Papio baboons (Castracane et al., 1981; Gesquiere et al., 2005). We found a significant effect of sex on hair cortisol in chacma baboons, with higher levels in females. Similar sex differences in hair cortisol were observed in olive baboons (Fourie, unpublished data), and although the sample size was too small for statistical comparison, female Guinea baboons also appeared to have higher hair cortisol concentrations than males (Fig. 4). This may be a taxonspecific characteristic of the Papio baboons reflecting either or both a genetic physiological difference between the sexes (e.g., differences in corticosteroid-binding globulin binding capacity Klosterman et al. 1986), perhaps related to the comparatively greater energetic and physiological 
demands of female reproduction (cortisol is known to be elevated during pregnancy - Klosterman et al., 1986), or differences in socio-behavioral effects on stress. Further research is needed to explore these intriguing sex differences in cortisol in the Papio baboons.

A survey of serum cortisol across several primate species reported a number of cases where there was a significant difference between males and females, but the nature of the differences appeared to be species-specific (Klosterman et al., 1986). Female cortisol levels exceeded male levels in captive vervet monkeys (Laudenslager et al., 2012); this trend was not observed in wild vervet monkeys (Fourie et al., 2015b). Bernstein et al. (2009) also report a non-significant trend of higher fecal cortisol in male langurs. No systematic gender differences in cortisol have been reported in humans (e.g., Kiess et al., 1995; Soldin et al., 2005), although Raul et al. (2004) reported a trend of higher hair cortisol concentration in men compared to women (also see Bechshøft et al., 2010 on polar bears).

Many studies have shown the potential of hair cortisol analysis to track physiological stress in individual animals (Fairbanks et al., 2011; Carlitz et al., 2014) and animal populations (Martin and Réale, 2008; Macbeth et al., 2010; Fourie et al., 2015a,b). Our results reflect similar individual chronic stress effects as evidenced by the wild, young adult dispersing male chacma baboon (Tsh1 in Fig. 4b) who took up residence in the city of Pretoria, Tshwane, South Africa

(http://mg.co.za/article/2006-03-17-east-rand-baboon-still-evades-captors). Local authorities actively pursued this individual for several months before he was captured, sedated and relocated. Thus, this particular animal was known to have experienced an extended period of intense stress before sampling, and his unusually high cortisol levels reflected this stress.

Hair steroid analysis presents a powerful and still largely underutilized tool in animal research. The technique is especially suited to address questions about chronic and basal stress hormone levels as hair archives uniquely averaged cortisol levels. The nature of the hair matrix makes it an especially stable environment for steroids and means that hair can be transported and stored under non-specific 
conditions. This, along with the use of remote techniques for collecting hair in wild and captive animals (Valderrama et al., 1999) makes hair highly suited for use in the field. However, careful project design and measured interpretation of results, based on the issues presented here, is essential. The best collection and sample preparation methods need to be decided on and standardized given the practical constraints of the project (also see Russell et al., 2015). Sampling error should be considered in the context of the research question and practical constraints, and a balance should be found. This can be done by determining the impact of anatomical site and hair characteristics on hair cortisol variation for a given species. Captive populations, if they can be investigated, may be useful for this purpose. Practical constraints may preclude sampling the same anatomical region or always getting large numbers of hairs, especially if remote sampling methods are used. Modifying the research question, or study design (e.g., increasing sample sizes) and effect-size thresholds can successfully adjust for increased variability in the data due to sampling effects. Many kinds of biological information are captured in hair cortisol (e.g., age, sex, individual experience); these can be the focus of interest but can also be confounding factors if not controlled for.

In sum, hair cortisol analysis is a relatively new, exciting and powerful tool in the study of physiology and stress in both wild and captive animals, and like any method has its own advantages, limitations and technical concerns, all of which need to be considered in order for it to be deployed to effectively address research questions.

\section{Acknowledgements}

We would like to thank Jean-Yves Robert and Dr. Benoit Quintard from the Museum de Besançon for arranging for the collection of Papio papio samples. We would like to thank Dr. Antoinette Kotze, Dr. Adrian Tordif and Mark Howitt from the National Zoological Gardens in South Africa for providing wild Papio ursinus samples from their collections and projects, and the Tanzanian National Parks Authority (TANAPA) for providing permission to conduct research in Mikumi 
366 National Park. We would like to thank the San Diego Zoo for its support in this research. We would

1 also like to thank Dr. Rui Diogo for allowing the collection of hair samples in the course of his research. We would also like to thank Sarah Putman from the Smithsonian's Conservation Research

Center for her support and advice. We would like to thank Michael Holland for his assistance in the laboratory. The research was supported with funds provided by the Center for the Advanced Study of Hominid Paleobiology at the George Washington University and through a Lewis Cotlow Grant. The opinions expressed herein and the interpretation and reporting of these data are the responsibility of the authors and in no way should be seen as an official recommendation, interpretation, or policy of the National Institutes of Health or the United States Government. The research presented in this paper was conducted as part of Dr. Fourie's doctoral research at the George Washington University before joining the NINR. 
6

382

383 11

\section{References}

Accorsi, P.A., Carloni, E., Valsecchi, P., Viggiani, R., Gamberoni, M., Tamanini, C., Seren, E., 2008. Cortisol determination in hair and faeces from domestic cats and dogs. Gen. Comp. Endocrinol. 155, 398-402.

Anielski, P., 2008. Hair analysis of anabolic steroids in connection with doping control - results from horse samples. J. Mass. Spectrom. 43, 1001-1008.

Bahr, N.I., Palme, R., Möhle, U., Hodges, J.K., Heistermann, M., 2000. Comparative aspects of the metabolism and excretion of cortisol in three individual nonhuman primates. Gen. Comp. Endocrinol. 117, 427-438.

Bechshøft, T.Ø., Sonne, C., Dietz, R., Born, E.W., Novak, M.A., Henchey, E., Meyer, J.S., 2010. Cortisol levels in hair of East Greenland polar bears. Sci. Total. Environ. 409, 831-834.

Beehner, J.C., Gesquiere, L., Seyfarth, R.M., Cheney, D.L., Alberts, S.C., Altmann, J., 2009. Testosterone related to age and life-history stages in male baboons and geladas. Horm. Behav. $56,472-480$.

Bennett, A., Hayssen, V., 2010. Measuring cortisol in hair and saliva from dogs: coat color and pigment differences. Domest. Anim. Endocrinol. 39, 171-180.

Bernstein, R., Nadler, T., Brown, J., Fourie, N.H., 2009. Variation in fecal glucocorticoid concentration in captive red-shanked douc langurs (Pygathrix nemaeus). Viet. J. Primatol. 1, 6574.

Carlitz, E.H.D., Kirschbaum, C., Stalder, T., van Schaik, C.P., 2014. Hair as a long-term retrospective cortisol calendar in orang-utans (Pongo spp.): new perspectives for stress monitoring in captive management and conservation. Gen. Comp. Endocrinol. 195, 151-156.

Carlitz, E.H.D., Kirschbaum, C., Miller, R., Rukundo, J., van Schaik, C.P., 2015. Effects of body region and time on hair cortisol concentrations in chimpanzees (Pan troglodytes). Gen. Comp. Endocrinol. 223, 9-15. 
Castracane, V., Cutler, G.B., Loriaux, D.L., 1981. Pubertal endocrinology of the baboon: adrenarche. A. J. Physiol. 241, E 305-309.

Chrousos, G.P., 2000. Stress, chronic inflammation, and emotional and physical wellbeing: concurrent effects and chronic sequelae. J. Allergy Clin. Immunol. 106, 275-291.

Cone, J., 1996. Mechanisms of drug incorporation into hair. Therap. Drug Monitor. 18, 438-443.

Davenport, M.D., Tiefenbacher, S., Lutz, C.K., Novak, M.A., Meyer, J.S., 2006. Analysis of endogenous cortisol concentrations in the hair of rhesus macaques. Gen. Comp. Endocrinol. 147, $255-261$.

de Kloet, E.R., Oitzl, M.S., Joëls, M., 1999. Stress and cognition: are corticosteroids good or bad guys? Trends Neurosci. 22, 422-426.

Fairbanks, L.A., Jorgensen, M.J., Bailey, J.N., Breidenthal, S.E., Grzywa, R., Laudenslager, M.L., 2011. Heritability and genetic correlation of hair cortisol in vervet monkeys in low and higher stress environments. Psychoneuroendocrinology 36, 1201-1208.

Fourie, N.H., Bernstein, R.M., 2011. Hair cortisol levels track phylogenetic and age related differences in hypothalamic-pituitary-adrenal (HPA) axis activity in non-human primates. Gen. Comp. Endocrinol. 174, 150-155.

Fourie, N.H., Jolly, C.J., Phillips-Conroy, J.E., Brown, J.L., Bernstein, R.M., 2015a. Variation of hair cortisol concentrations among wild populations of two baboon species (Papio anubis, P. hamadryas) and a population of their natural hybrids. Primates 56, 259-272.

Fourie, N.H., Turner, T.R, Brown, J.L., Pampush, J.D., Lorenz, J.G., Bernstein, R.M., $2015 b$. Variation in vervet (Chlorocebus aethiops) hair cortisol concentrations reflects ecological disturbance by humans. Primates 56, 365-373.

Gaillard, Y., Vayssette, F., Pépin, G., 2000. Compared interest between hair analysis and urinalysis in doping controls: results for amphetamines, corticosteroids and anabolic steroids in racing cyclists. Forensic Sci. Int. 107, 361-379. 
Gao, X., Alvo, M., Chen, J., Li, G., 2008. Nonparametric multiple comparison procedures for unbalanced one-way factorial designs. J. Stat. Plan. Infer. 77, 2574-2591.

Gesquiere, L., Altmann, J., Khan, M., Couret, J., Yu, J., Endres, C., Lynch, J., Ogola, P., Fox, E., Alberts, S., Wango, E., 2005. Coming of age: steroid hormones of wild immature baboons (Papio cynocephalus). Am. J. Primatol. 67, 83-100.

Harkey, M.R., 1993. Anatomy and physiology of hair. Forensic Sci. Int. 63, 9-18.

Kalra, S., Einarson, A., Karaskov, T., van Uum, S., Koren, G., 2012. The relationship between stress and hair cortisol in healthy pregnant women. Clin. Invest. Med. 30, E 103-107.

Kiess, W., Meidert, A., Dressendörfer, R.A., Schriever, K., Kessler, U., König, A., Schwarz, H.P., Strasburger, C.J., 1995. Salivary cortisol levels throughout childhood and adolescence: relation with age, pubertal stage, and weight. Pediatr. Res. 37, 502-506.

Kirschbaum, C., Tietze, A., Skoluda, N., Dettenborn, L., 2009. Hair as a retrospective calendar of cortisol production - Increased cortisol incorporation into hair in the third trimester of pregnancy. Psychoneuroendocrinology 34, 32-37.

Klosterman, L.L., Murai, J.T., Siteri, P.K., 1986. Cortisol levels, binding, and properties of corticosteroid-binding globulin in the serum of primates. Endocrinology 118, 424-434.

Konietschke, F., 2015. nparcomp: Perform Multiple Comparisons and Compute Simultaneous Confidence Intervals for the Nonparametric Relative Contrast Effects. R package version 2.6, http://CRAN.R-project.org/package=nparcomp.

Koren, L., Mokady, O., Karaskov, T., Klein, J., Koren, G., Geffen, E., 2002. A novel method using hair for determining hormonal levels in wildlife. Anim. Behav. 63, 403-406.

Koren, L., Mokady, O., Geffen, E., 2008. Social status and cortisol levels in singing rock hyraxes. Horm. Behav. 54, 212-216. 
452 Laudenslager, M.L., Jorgensen, M.J., Fairbanks, L.A., 2012. Developmental patterns of hair cortisol 1 in male and female nonhuman primates: lower hair cortisol levels in vervet males emerge at

Macbeth, B.J., Cattet, M.R.L., Stenhouse, G.B., Gibeau, M.L., Janz, D.M., 2010. Hair cortisol concentration as a noninvasive measure of long-term stress in free-ranging grizzly bears (Ursus arctos): considerations with implications for other wildlife. Can. J. Zool. 88, 935-949.

Martin, J.G., Réale, D., 2008. Animal temperament and human disturbance: implications for the response of wildlife to tourism. Behav. Processes 77, 66-72.

Melecchi, M.I.S., Martinez, M.M., Abad, F.C., Zini, P.P., do Nascimento Filho, I., Caramão, E.B., 2002. Chemical composition of Hibiscus tiliaceus L. flowers: a study of extraction methods. J. Sep. Sci. 25, 86-90.

Nelson, R., 2005. An Introduction to Behavioral Endocrinology. Sinauer Associates, Sunderland, MA.

Pearson, B.L., Reeder, D.M., Judge, P.G., 2015. Crowding increases salivary cortisol but not selfdirected behavior in captive baboons. Am. J. Primatol. 77, 462-467.

Phillips-Conroy, J.E., Jolly, C.J., 1988. Dental eruption schedules of wild captive baboons. Am. J. Primatol. 15, 17-29.

Phillips-Conroy, J.E., Jolly, C.J., Rogers, J.A., 1987. Hematocrits of free-ranging baboons: variation within and among populations. J. Med. Primatol. 16, 389-402.

R Development Core Team, 2015. R: A Language and Environment for Statistical Computing. R Foundation for Statistical Computing, Vienna, Austria, https://www.R-project.org/

Raul, J.-S., Cirimele, V., Ludes, B., Kintz, P., 2004. Detection of physiological concentrations of cortisol and cortisone in human hair. Clin. Biochem. 37, 1105-1111. 
Russell, E., Koren, G., Rieder, M., Van Uum, S., 2012. Hair cortisol as a biological marker of chronic stress: current status, future directions and unanswered questions.

Psychoneuroendocrinology 37, 589-601.

Russell, E., Kirschbaum, C., Laudenslager, M.L., Stalder, T., de Rijke, Y., van Rossum, E.F., Van Uum, S., Koren, G., 2015. Toward standardization of hair cortisol measurement: results of the first international interlaboratory round robin. Ther. Drug. Monit. 37, 71-75.

Saltzman, W., Schultz-Darken, N.J., Scheffler, G., Wegner, F.H., Abbott, D.H., 1994. Social and reproductive influences on plasma cortisol in female marmoset monkeys. Physiol. Behav. 56, 801-810.

Sapolsky, R.M., 1992a. Stress, the Aging Brain and the Mechanisms of Neuron Death. MIT Press, Cambridge, MA.

Sapolsky, R.M., 1992b. Cortisol concentrations and the social significance of rank instability among wild baboons. Psychoneuroendocrinology 17, 701-709.

Sapolsky, R.M., 2004. Why Zebras Don't Get Ulcers: A Guide to Stress, Stress Related Diseases And Coping. W.H. Freeman, New York.

Sapolsky, R.M., 2005. The influence of social hierarchy on primate health. Science 308, 648-652.

Sauve, B., Koren, G., Walsh, G., Tokmakejian, S., van Uum, S.H.M., 2007. Measurement of cortisol in human hair as a biomarker of systemic exposure. Clin. Invest. Med. 30, E 183-191.

Schinor. E.C., Salvador, M.J., Turatti, I.C.C., Zucchi, O.L.A.D., Dias, D.A., 2004. Comparison of classical and ultrasound-assisted extractions of steroids and triterpenoids from three Chresta spp. Ultrason. Sonochem. 11, 415-421.

Sharpley, C., Kauter, K., McFarlane, J., 2010a. An investigation of hair cortisol concentration across body sites and within hair shaft. Clin. Med. Insights: Endocrinol. Diabet. 3, 17-23. 
Sharpley, C., Kauter, K., McFarlane, J., 2010b. Hair cortisol concentration differs across site and person: localization and consistency of responses to a brief pain stressor. Phyiol. Res. 59, 979983.

Soldin, O.P., Hoffman, E.G., Waring, M.A., Soldin, S.J., 2005. Pediatric reference intervals for FSH, LH, estradiol, T3, free T3, cortisol, and growth hormone on the DPC IMMULITE 1000. Clin. Chim. Acta 355, 205-210.

Terwissen, C.V., Mastromonaco, G.F., Murray, D.L., 2013. Influence of adrenocorticotrophin hormone challenge and external factors (age, sex, and body region) on hair cortisol concentration in Canada lynx (Lynx canadensis). Gen. Comp. Endocrinol. 194, 162-167.

Thomson, S., Koren, G., Fraser, L.A., Rieder, M., Friedman, T.C., van Uum, S.H.M., 2010. Hair analysis provides a historical record of cortisol levels in Cushing's syndrome. Exp. Clin. Endocrinol. Diabetes 118, 133-138.

Valderrama, X., Karesh, W.B., Wildman, D.E., Melnick, D.J., 1999. Noninvasive methods for collecting fresh hair tissue. Mol. Ecol. 8, 1749-1752.

Webb, E., Thomson, S., Nelson, A., White, C., Koren, G., Rieder, M., 2010. Assessing individual systemic stress through cortisol analysis of archaeological hair. J. Archaeol. Sci. 37, 807-812.

Webb, E.C., White, C.D., Van Uum, S., Longstaffe, F.J., 2015. Integrating cortisol and isotopic analyses of archeological hair: reconstructing individual experiences of health and stress. Am. J. Phys. Anthropol. 156, 577-594.

Yamanashi, Y., Morimura, N., Mori, Y., Hayashi, M., Suzuki, J., 2013. Cortisol analysis of hair of captive chimpanzees (Pan troglodytes). Gen. Comp. Endocrinol. 194, 55-63. 
5¥2 Table 1. Estimates of the hair growth rates and time to full regrowth of hair for six zoo-kept primate 5 species.

\begin{tabular}{ccccccc} 
Species & $\mathbf{n} \begin{array}{c}\text { Estimated Average } \\
\text { Growth Rate } \\
\text { (cm/month) }\end{array}$ & SD $\begin{array}{c}\text { Average Length } \\
\text { (cm) }\end{array}$ & SD & $\begin{array}{c}\text { Estimated Time } \\
\text { Archived } \\
\text { (months) }\end{array}$ \\
\hline A. nigroviridis & 2 & 1.7 & 0.3 & 5.8 & 1.0 & 3.4 \\
C. chrysogaster & 3 & 0.7 & 0.1 & 5 & 1.0 & 7.1 \\
T. gelada & 1 & 3.5 & & 12 & 0.6 & 3.4 \\
G. gorilla & 3 & 2.7 & 2.7 & 7.5 & 0.7 & 2.8 \\
H. gabriellae & 1 & 0.8 & & 4.6 & 6.5 & 5.8 \\
S. syndactylus & 1 & 1 & & 5.1 & 0.5 & 5.1 \\
\hline
\end{tabular}




\section{Figure captions}

Fig. 1. Mean and standard error plot showing that incubation in a sonicating hot water bath increases the amount of cortisol extracted from hair. All extractions that were incubated in a sonicating hot water bath yielded more cortisol than samples extracted under ambient conditions.

Fig. 2. Inter-aliquot variance of white-faced saki monkey hair samples consisting of different numbers of hairs ( $3 \mathrm{mg}=21$ hairs, $10 \mathrm{mg}=71$ hairs and $20 \mathrm{mg}=144$ hairs $)$ per aliquot.

Fig. 3. Plot showing significantly higher cortisol concentrations were sequestered in hair from the thigh region compared to hair from the base of the tail and shoulder regions in five adult female yellow baboons.

Fig. 4. Scatter plot with Lowess regression fitted to the data showing the age-related decline in hair cortisol levels in both male and female Guinea baboons.

Fig. 5. Box plot showing the age-related decline in hair cortisol levels in both male and female chacma baboons. Female chacma baboons had higher hair cortisol levels than males. Note the high hair cortisol level of the adult male Tsh1 that was captured after an extended pursuit over several weeks in the city of Pretoria. 
Figures

Fig. 1.

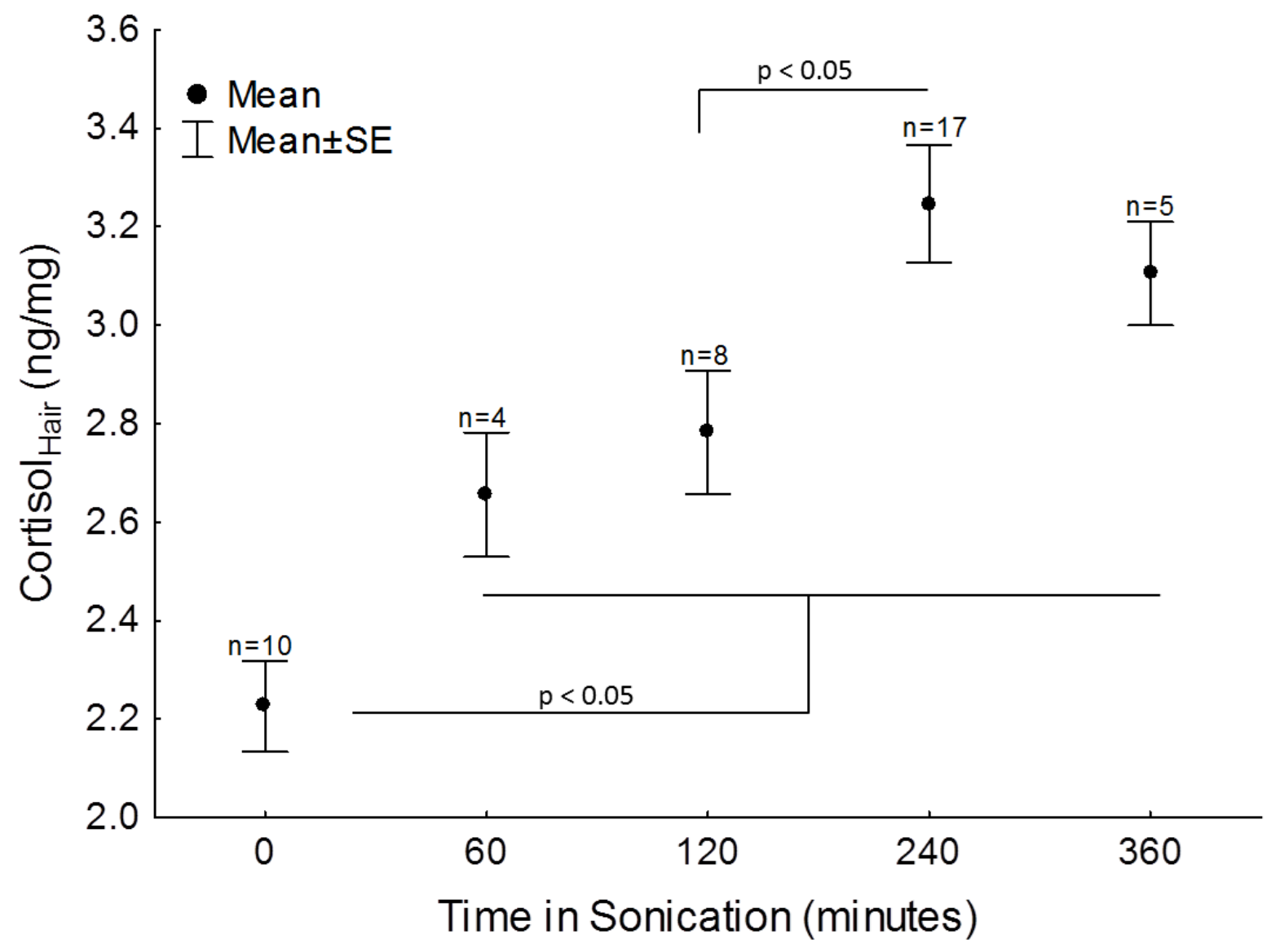


Fig. 2.

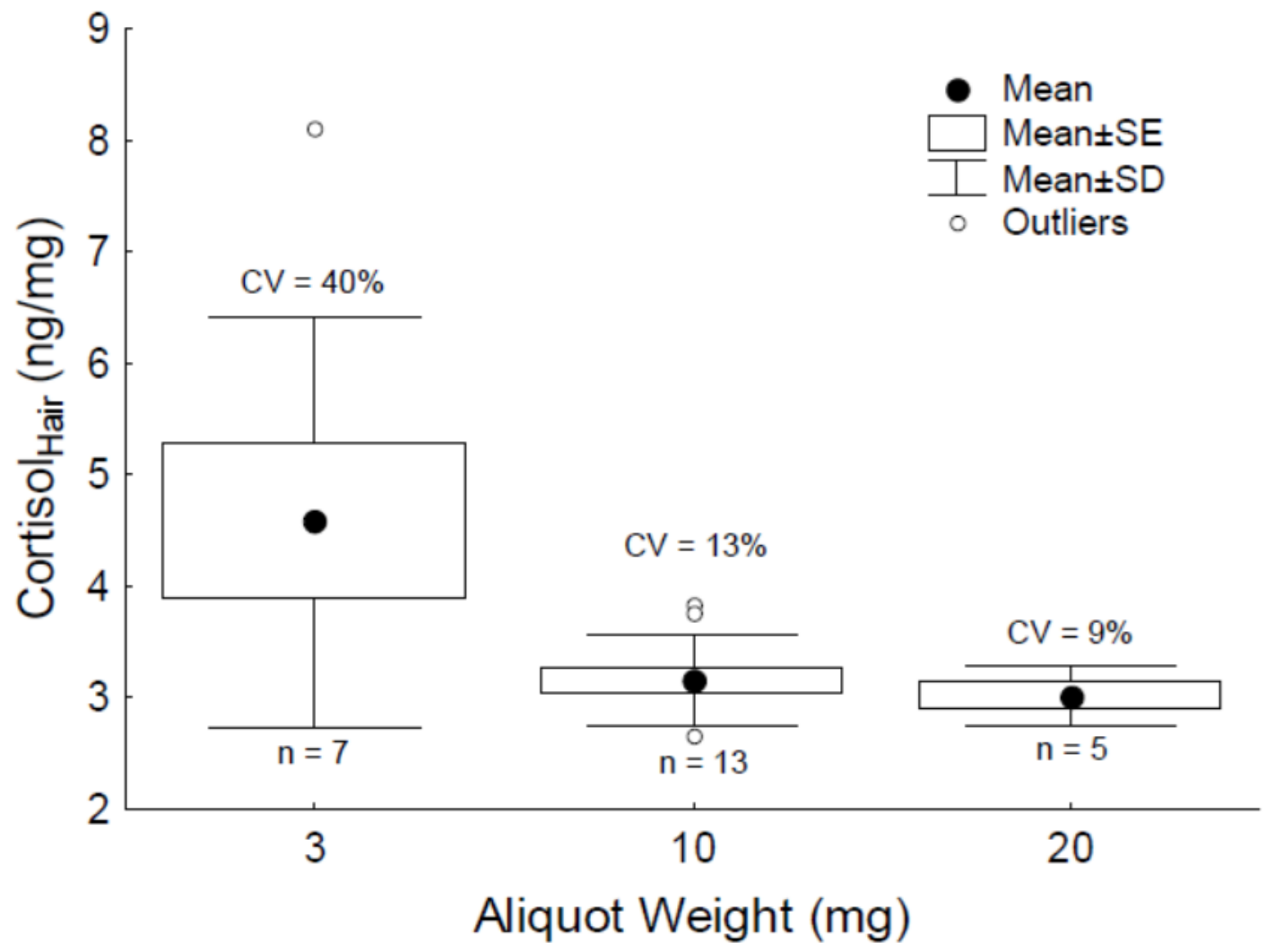


Fig. 3.

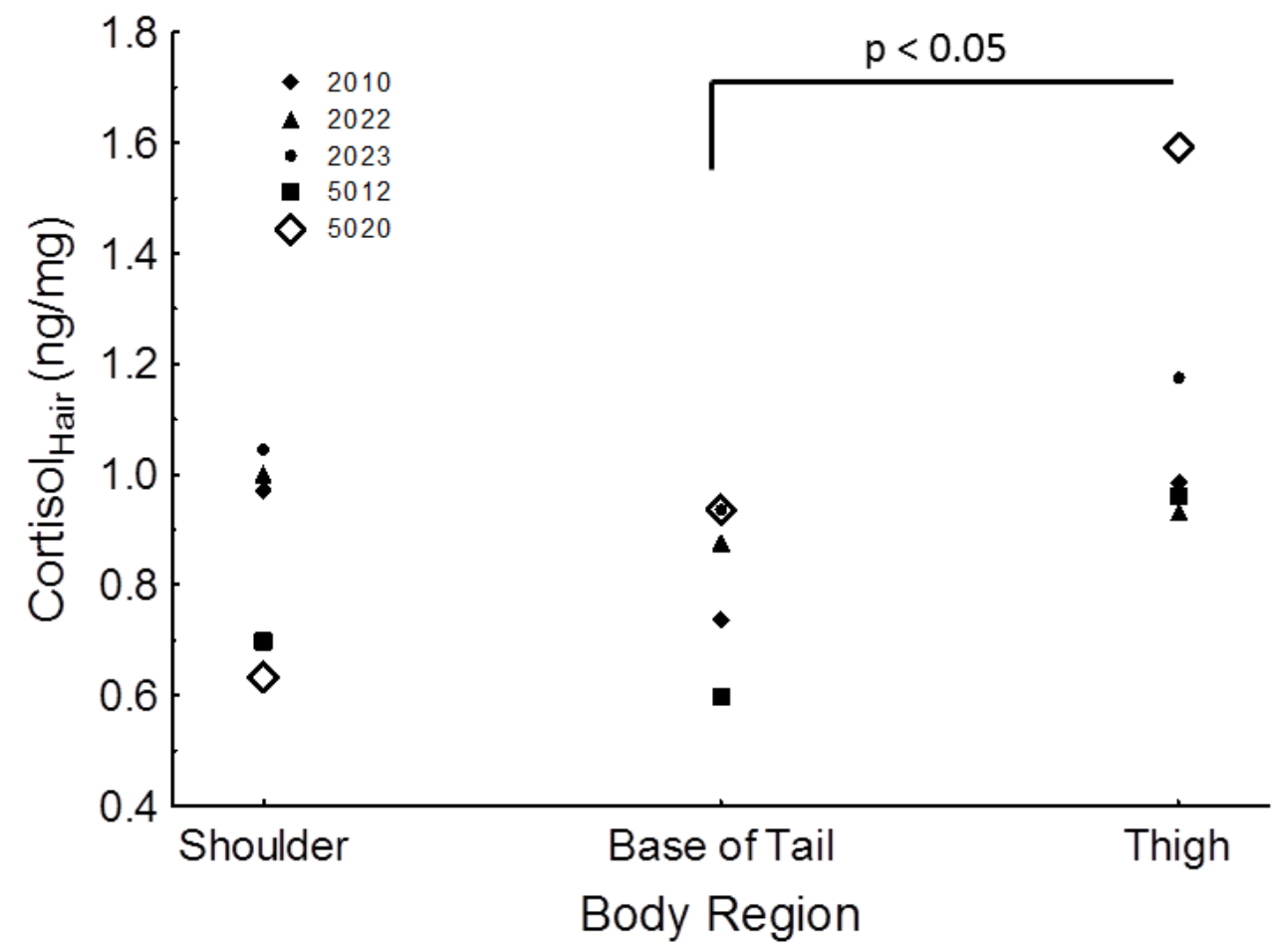


Fig. 4.

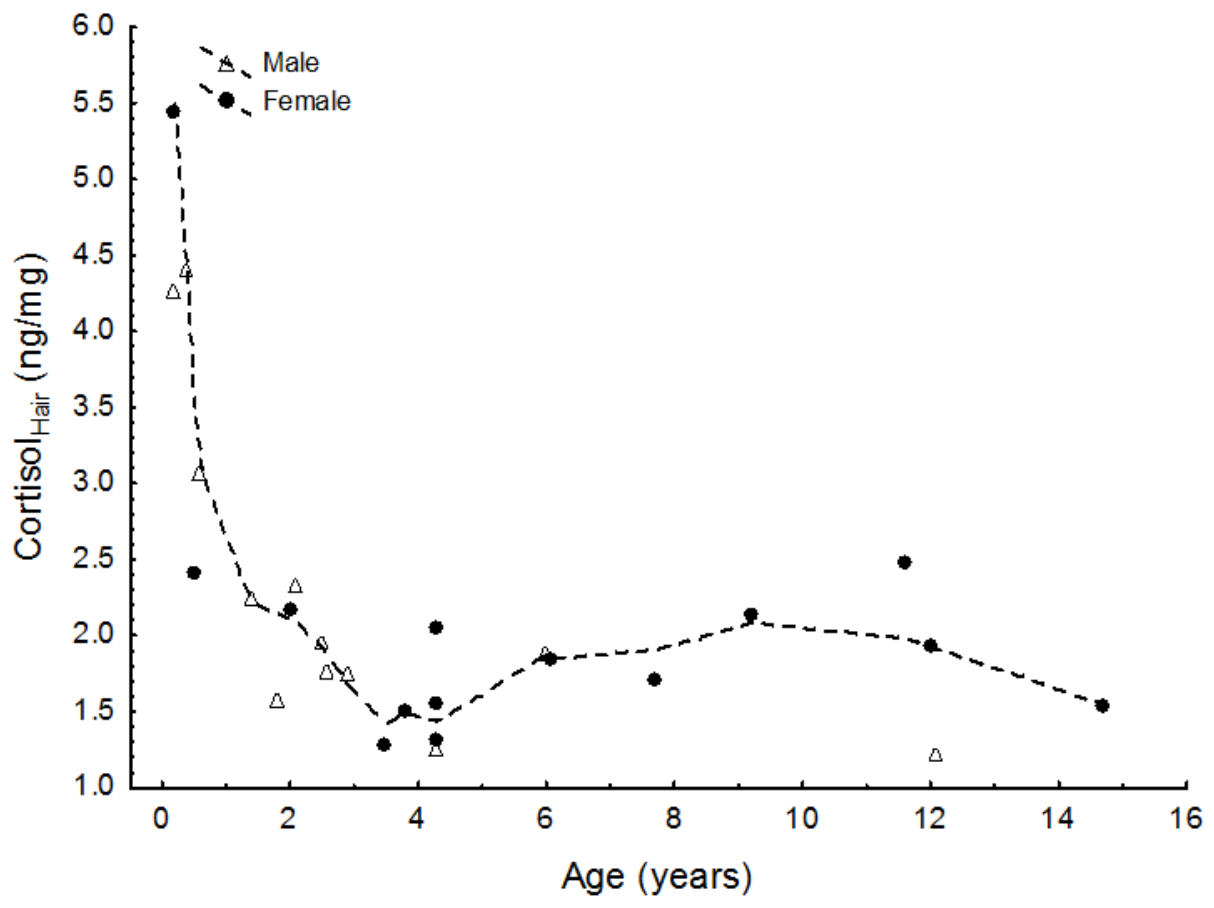


Fig. 5.

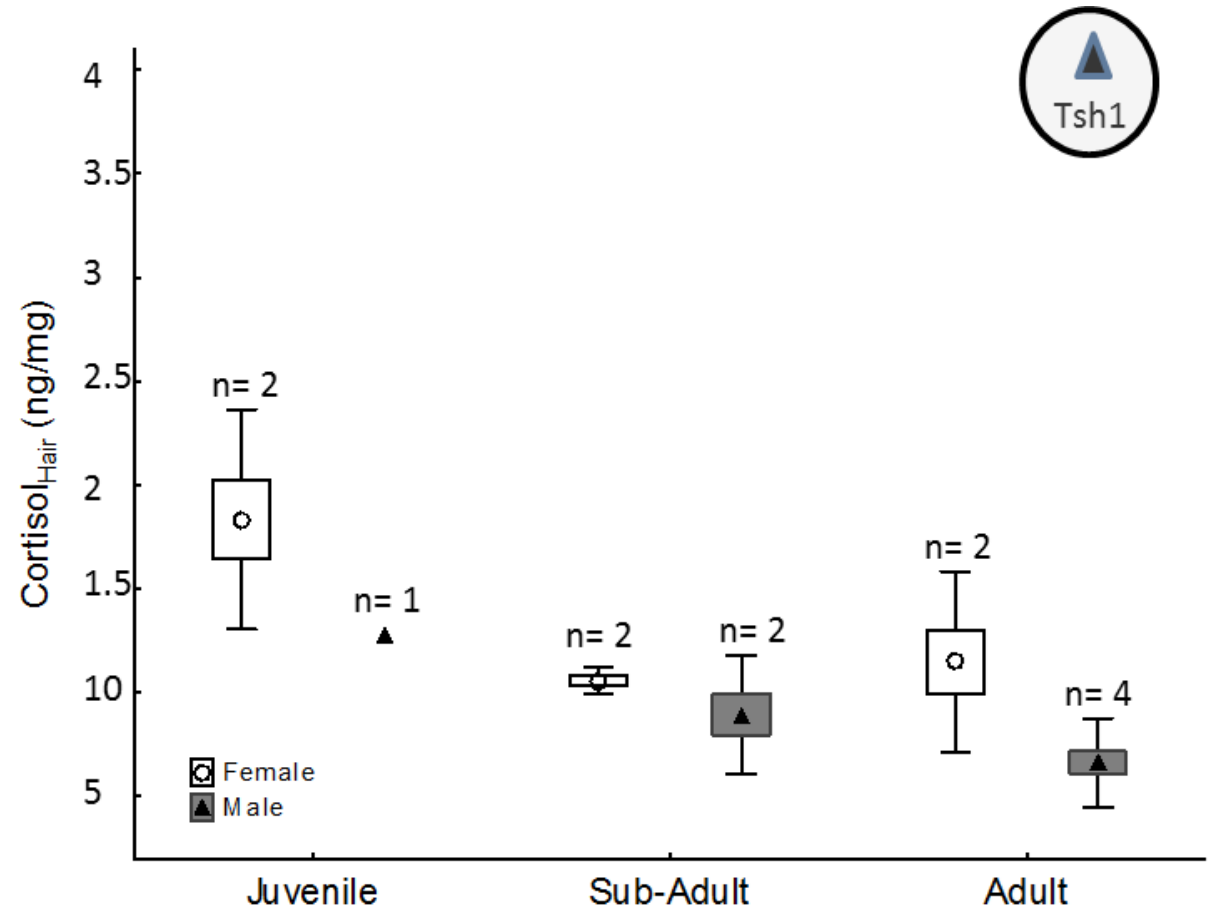

